\title{
An eight-long non-coding RNA signature as a candidate prognostic biomarker for lung cancer
}

\author{
ZHENBO TU $^{1 *}$, DU HE$^{2^{*}}$, XINZHOU DENG ${ }^{1}$, MENG XIONG $^{1}$, XIAOXING HUANG $^{1}$, \\ XINRAN LI ${ }^{1}$, LING HAO ${ }^{1}$, QIANSHAN DING ${ }^{3}$ and QIUPING ZHANG ${ }^{1}$ \\ ${ }^{1}$ Department of Immunology, Wuhan University School of Basic Medical Sciences, Wuhan, Hubei; \\ ${ }^{2}$ Department of Oncology, The Central Hospital of Enshi Autonomous Prefecture, \\ Enshi Clinical College of Wuhan University, Enshi, Hubei; ${ }^{3}$ Department of Gastroenterology, \\ Renmin Hospital of Wuhan University, Wuhan, Hubei, P.R. China
}

Received January 13, 2016; Accepted February 22, 2016

DOI: $10.3892 /$ or.2016.4817

\begin{abstract}
Cumulative evidence suggests that long non-coding RNAs (lncRNAs) may be good biomarkers in various types of tumors. In the present study, we mined lncRNA expression profiling in 739 lung cancer patients from Gene Expression Omnibus (GEO) datasets. A risk score model was constructed based on the expression data of these eight lncRNAs in the training dataset (GSE30219). The validation for the association was performed in three independent testing sets (GSE31210, GSE37745 and GSE19188). Finally, a set of eight lncRNA genes (AK021595, BC030759, AK000053, AK124307, BC020384, AK022024, CR615992 and AF085995) were identified by the random survival forest algorithm. Using a risk score based on the expression signature of these lncRNAs, we separated the patients into low-risk and high-risk groups with significantly different survival times in the training set. This finding was validated in the other three testing sets. Further study revealed that the eight-lncRNA expression signature was independent of age and gender. Gene Set Enrichment Analysis (GSEA) suggested that lncRNAs were involved in cell cycle
\end{abstract}

Correspondence to: Professor Qiuping Zhang, Department of Immunology, School of Basic Medical Sciences, Wuhan University, 185 Donghu Road, Wuchang, Wuhan, Hubei 430071, P.R. China

E-mail: qpzhang@whu.edu.cn

Dr Qianshan Ding, Department of Gastroenterology, Renmin Hospital of Wuhan University, Wuhan, Hubei, P.R. China

E-mail: iamdqs@163.com

*Contributed equally

Abbreviations: lncRNA, long non-coding RNA; GEO, Gene Expression Omnibus; GSEA, Gene Set Enrichment Analysis; KEGG, Kyoto Encyclopedia of Genes and Genomes

Key words: long non-coding RNA, lung cancer, survival, gene signature and DNA replication signaling pathways. Therefore, the eight lncRNAs may be candidate prognostic biomarkers for lung cancer patients.

\section{Introduction}

Lung cancer is one of the most commonly diagnosed cancers and remains the leading cause of cancer-related death worldwide $(1,2)$. It has been traditionally subdivided into two principal groups, namely, small cell lung cancer and non-small cell lung cancer. The latter type is more common than the former. Despite diverse treatment methods including surgery, radiation therapy and chemotherapy, the overall 5-year survival rate remains $\sim 18.2 \%$ (3). The high mortality rate of lung cancer is partly due to the lack of effective prognostic biomarkers. Therefore, the identification of novel prognostic factors as biomarkers that may be used in the early detection of lung cancer is critical.

Long non-coding RNAs (lncRNAs) are mRNA-like transcripts with more than 200 nucleotides that lack significant protein-coding abilities $(4,5)$. Increasing evidence suggests that IncRNAs are a new class of players involved in the development and progression of cancer (6). More and more research suggests that these transcripts are frequently aberrantly expressed in cancers, and some have been implicated in the diagnosis and prognostication (7) in neuroblastoma (8), prostate (9), breast $(10-12)$, ovarian $(13,14)$, gastric (15) and colorectal cancer $(16,17)$, and multiple myeloma (18). Due to the specific expression of lncRNAs in cancer, lncRNAs could become biomarkers by which to diagnosis cancer or predict patient survival. Thus, identification of various lncRNAs which are specifically expressed in lung cancer may have predictive and prognostic value for lung cancer patients.

Currently, massive lncRNA-specific probes are presented on microarray platforms (Affymetrix U133 Plus 2.0); thus, we are able to use previously published gene expression microarray data from the Gene Expression Omnibus (GEO) database to identify various prognostic signature lncRNAs. Furthermore, bioinformatic analysis was used to identify the signaling pathways that involve lncRNAs by Gene Set Enrichment Analysis (GSEA). 


\section{Materials and methods}

Lung cancer datasets and patient information. Lung cancer datasets were downloaded from the GEO database. A total of 739 patients were utilized in the present study after filtering out samples without clinical survival information. It included 293 patients from GSE30219 (19), 226 patients from GSE31210 (20), 168 patients from GSE37745 (21), and 52 patients from GSE19188 (22). We selected these datasets that included $>50$ patients with survival status information. We followed the strategy of using the largest dataset (GSE30219) as training set. Three independent datasets (GSE31210, GSE37745 and GSE19188) were included in the present study as testing sets.

Microarray processing and lncRNA profile mining. All the microarray raw data (CEL files) of four lung cancer cohorts were processed using the robust multichip average (RMA) algorithm for background adjustment (23). GATExplorer was used to process microarrays on a local computer for gene expression of lncRNAs (24). lncRNA mapper was obtained from GATExplorer, which included the probes that do not map to any coding region but that were mapped to a database for non-coding RNAs of human and mouse (derived from RNAdb (25). The coding potential analysis of the lncRNAs was carried out by CNCI to classify protein-coding or non-coding transcripts (26). Each lncRNA included at least a minimum of three probes mapping in the corresponding lncRNA entity. We created a risk-score formula according to the expression of these eight lncRNAs for survival prediction. Patients having higher risk scores were expected to have poorer survival outcomes.

Statistical analysis. The association between the lncRNA gene expression and patient survival was assessed by univariable Cox proportional hazards regression analysis along with a permutation test using BRB-ArrayTools (Biometric Research Branch) package (27) in the training set. We identified expression of several lncRNAs that were strongly correlated with survival. Considering that a smaller number of genes in the model would make the model more practical, we performed the random survival forests variable hunting (RSFVH) algorithm (28). Using a smaller number of genes selected fitted in a multivariable Cox regression model; we constructed a formula to predict survival in the training set. Each patient was assigned a risk score that is a linear combination of the expression levels of the significant lncRNAs weighted by their respective Cox regression coefficients (29). According to this risk score, patients in the training set were divided into low-risk and high-risk groups using the median risk score as the cut-off. The Kaplan-Meier method was used to estimate survival time, and other three independent testing groups were performed for validation. Differences in survival times between the lowrisk and high-risk groups in each set were compared by the two-sided log-rank test, respectively.

Bioinformatic analysis of IncRNA gene function. GSEA was performed by the JAVA program (http://www.broadinstitute. org/gsea) using MSigDB C2 CP: canonical pathway gene set collection $(1,320$ gene sets available). Gene sets with a false discovery rate (FDR) value $<0.05$ after performing 1,000 permutations were considered to be significantly enriched (30). Cytoscape (version 2.8.2) and the Enrichment Map software were used to visualize the GSEA results (31). Encyclopedia of Genes and Genomes (KEGG) enrichment analyses of the co-expressed protein-coding genes with prognostic IncRNAs were performed to predict the biological function of prognostic lncRNAs using the DAVID Bioinformatics Tool (version 6.7) (32). Enrichment analysis was carried out using the functional annotation clustering options, and was limited to KEGG pathways in the 'Biological Process' categories.

\section{Results}

Identification of prognostic IncRNA genes from the training set. As summarized in the workflow (Fig. 1), all analyses were performed in the training set (GSE30219) and validated in the testing set (GSE31210, GSE37745 and GSE19188). The training set $(n=293)$ was analyzed for the detection of prognostic lncRNA genes. By subjecting the lncRNA expression data derived from the training set to univariable Cox proportional hazards regression analysis using the BBRB-ArrayTools, we identified a set of $36 \operatorname{lncRNAs}$ that were strongly correlated with patient overall survival ( $p<0.001$ and FDR $<0.001$ ) from a total of 5,635 lncRNAs. Based on the random survival forests model (see Materials and methods), the eight lncRNAs (Table I) were selected as predictors (Fig. 2). In Table I a list of these eight lncRNAs is shown with their obtained coefficient and variable importance values. Based on these results, BC030759 was the most relevant with overall survival in the training set $(\mathrm{HR}=3.513)$; the positive coefficients of the lncRNAs (AK021595, BC030759, AK000053, BC020384 and AK022024) indicated that their higher levels of expression were associated with shorter survival, and the negative coefficients of the other lncRNAs (AK124307, CR615992 and AF085995) indicated that their higher levels of expression were associated with longer survival. All of the eight lncRNAs have been verified in the ncRNA Expression Database (www.nred.matticklab.com) and these eight transcripts were classified as ncRNAs in this website (33). As coding potential analysis is commonly used to classify whether a transcript is of coding potential or not (34), we used CNCI to test those eight transcripts (26). This tool also suggested that all the eight transcripts were non-coding transcripts with no coding potential.

An eight-IncRNA signature predicts survival of lung cancer patients in the training set. To investigate whether the eight-lncRNA signature could provide an accurate prediction of survival in lung cancer patients, we created a risk-score formula according to the expression of these eight lncRNAs for survival prediction in the training set GSE30219 $(n=293)$, as follows: risk score, $0.212 * \mathrm{AK} 021595+0.416 * \mathrm{BC} 030759$ $+0.322 *$ AK000053-0.165*AK124307+0.301*BC020384+0.42 3*AK022024-0.084*CR615992-0.459*AF085995. Then, we calculated the eight-lncRNA signature risk score for each patient in the training set. Patients were divided into a low-risk or high-risk group using the median risk score as cut-off value. Patients in the high-risk group had a shorter survival time than 


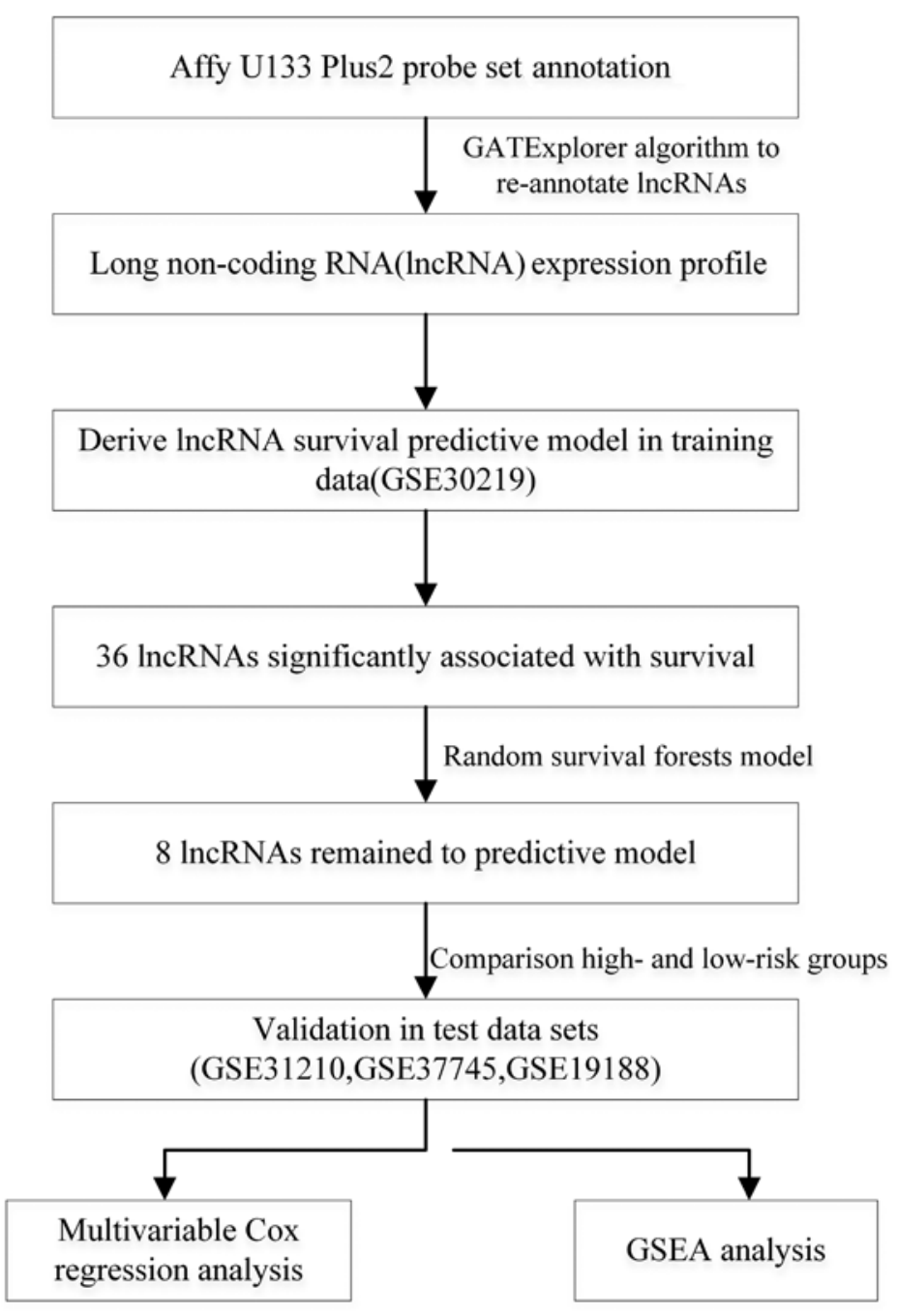

Figure 1. Diagram of the study. The order of analyses to obtain the large number of lncRNAs and develop the risk score model to predict prognostic outcomes by validation in the test data sets.
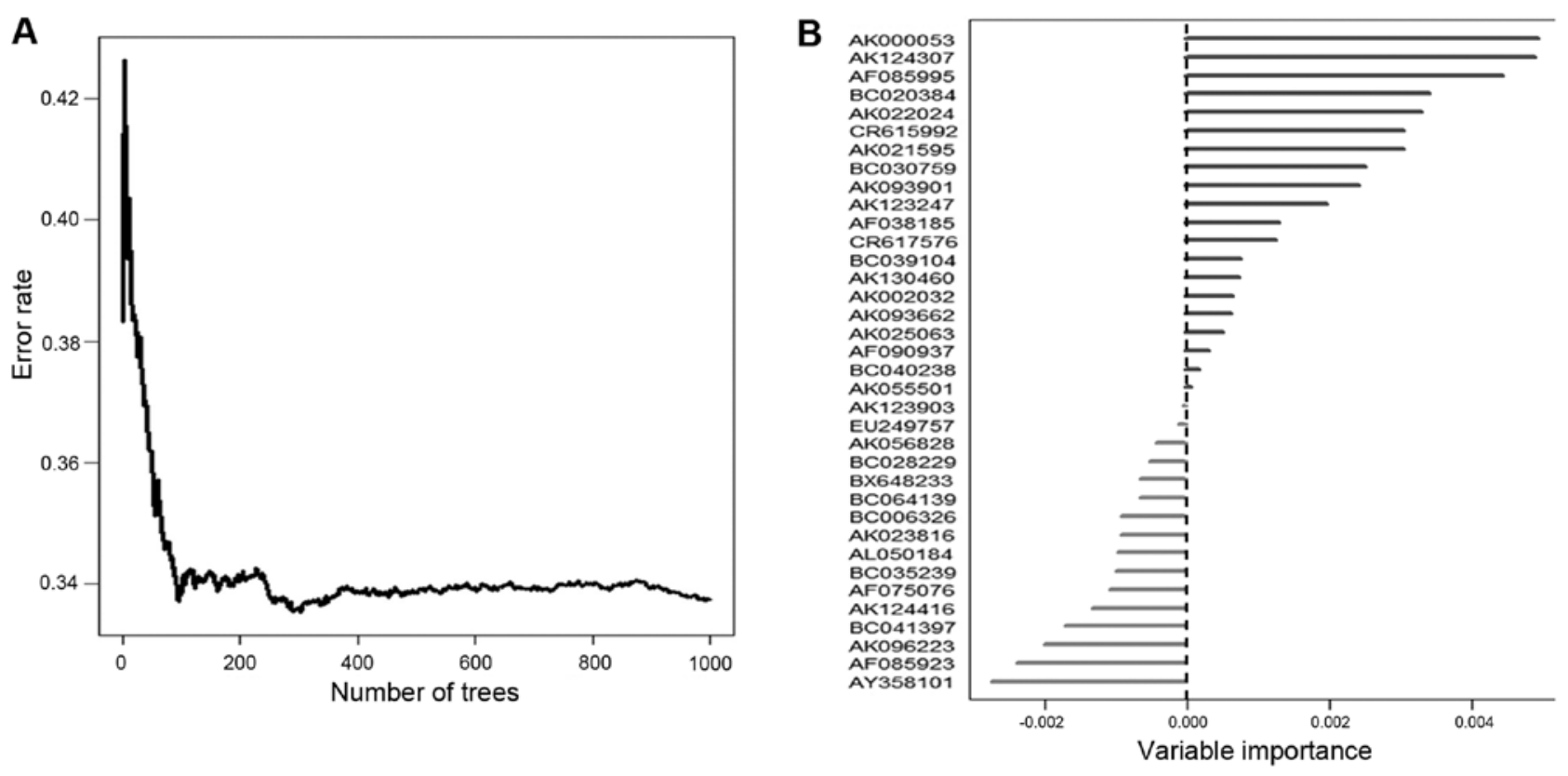

Figure 2. Error rate for the data as a function of trees (A) and out-of-bag importance values for predictors (B). 
A Training set (GSE30219), $\mathrm{n}=293$

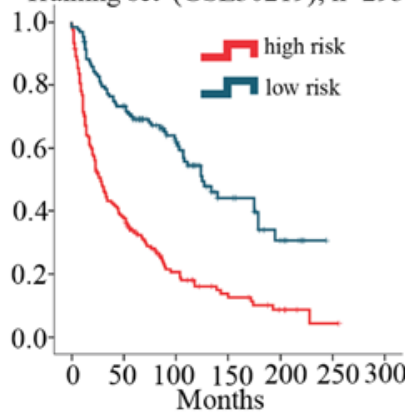

C Testing set (GSE37745), $\mathrm{n}=168$

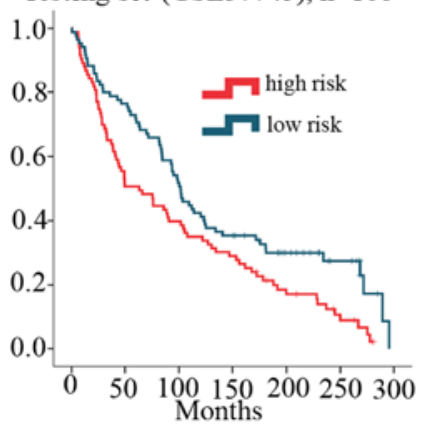

B Testing set (GSE31210), $\mathrm{n}=226$

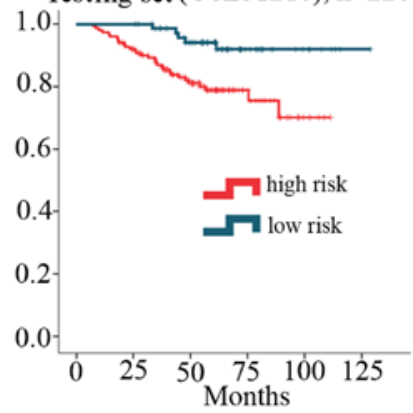

D Testing set (GSE19188), $\mathrm{n}=52$

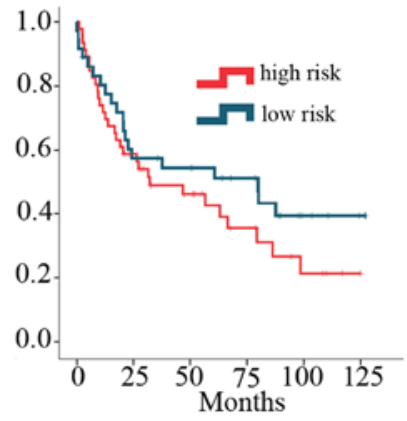

Figure 3. Kaplan-Meier estimates of the survival of GEO patients using the eight-lncRNA signature. The Kaplan-Meier plots were used to visualize the survival probabilities for the low-risk vs. high-risk group of GEO patients determined on the basis of the median risk score from the training set. (A) KaplanMeier curves for GSE30219 training-set patients (n=293). (B) Kaplan-Meier curves for GSE31210 testing-set patients (n=226). (C) Kaplan-Meier curves for GSE37745 testing-set patients $(n=168)$. The longest survival time in the model was 250 months, thus the patients whose survival time was $>300$ months was removed. (D) Kaplan-Meier curves for GSE19188 testing-set patients ( $\mathrm{n}=52)$. The tick marks on the Kaplan-Meier curves represent the censored subjects. The differences between the two curves were determined by the two-sided log-rank test.

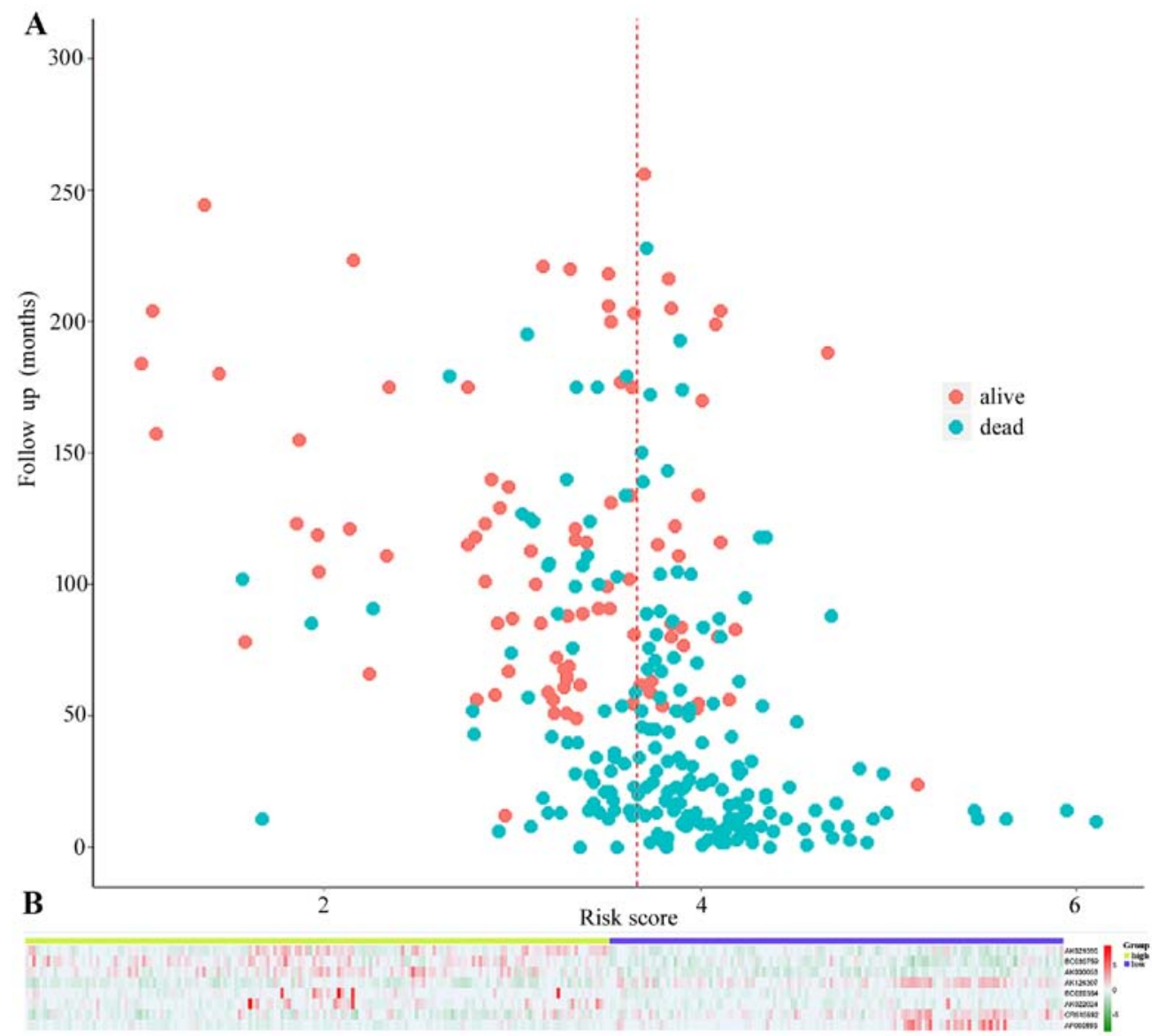

Figure 4. lncRNA risk score analysis of the GSE30219 patients. (A) Patient overall survival status. (B) Heatmap of the lncRNA expression profiles. Rows represent lncRNAs and columns represent patients. 
Table I. Eight lncRNAs are significantly associated with the overall survival in the training set $(n=293)$.

\begin{tabular}{|c|c|c|c|c|c|c|}
\hline lncRNA & $\begin{array}{c}\text { Parametric } \\
\text { p-value }\end{array}$ & FDR & $\begin{array}{l}\text { Hazard } \\
\text { ratio }\end{array}$ & Coefficient & $\begin{array}{c}\text { Variable } \\
\text { importance }\end{array}$ & $\begin{array}{c}\text { Relative } \\
\text { importance }\end{array}$ \\
\hline AK021595 & $3.00 \mathrm{E}-07$ & $8.90 \mathrm{E}-05$ & 1.621 & 0.212 & 0.003 & 0.6171 \\
\hline ВС030759 & $3.00 \mathrm{E}-07$ & 8.90E-05 & 3.513 & 0.416 & 0.0025 & 0.5086 \\
\hline AK000053 & $3.00 \mathrm{E}-07$ & $8.90 \mathrm{E}-05$ & 1.884 & 0.322 & 0.0049 & 1 \\
\hline AK 124307 & $6.00 \mathrm{E}-07$ & 0.000154 & 0.696 & -0.165 & 0.0049 & 0.9943 \\
\hline ВC020384 & $1.40 \mathrm{E}-06$ & 0.000303 & 1.716 & 0.301 & 0.0034 & 0.6914 \\
\hline AK022024 & 1.60E-06 & 0.000334 & 2.127 & 0.423 & 0.0033 & 0.6686 \\
\hline CR615992 & $2.60 \mathrm{E}-06$ & 0.000488 & 0.527 & -0.084 & 0.003 & 0.6171 \\
\hline AF085995 & $6.10 \mathrm{E}-06$ & 0.000764 & 0.458 & -0.459 & 0.0044 & 0.9029 \\
\hline
\end{tabular}

Websites of the eight lncRNAs are as follows:

1, AK021595 (http://www.genome.ucsc.edu/cgi-bin/hgc?hgsid=476093793_1SpaEOeLgD2BNxvEvsDDCbEI8hWx\&c=chr20\&o=33617949\& $\mathrm{t}=33619432 \& \mathrm{~g}=\mathrm{mrna} \& \mathrm{i}=\mathrm{AK} 021595)$

2, BC030759 (http://www.genome.ucsc.edu/cgi-bin/hgc?hgsid=476093793_1SpaEOeLgD2BNxvEvsDDCbEI8hWx\&c=chr1\&o=112961901\& $\mathrm{t}=112975292 \& \mathrm{~g}=\mathrm{mrna} \& \mathrm{i}=\mathrm{BC} 030759)$

3, AK000053 (http://www.genome.ucsc.edu/cgi-bin/hgc?hgsid=476093793_1SpaEOeLgD2BNxvEvsDDCbEI8hWx\&c=chrl\&o=108932526\& $\mathrm{t}=108934363 \& \mathrm{~g}=\mathrm{mrna} \& \mathrm{i}=\mathrm{AK} 000053)$

4, AK124307 (http://www.genome.ucsc.edu/cgi-bin/hgc?hgsid=476093793_1SpaEOeLgD2BNxvEvsDDCbEI8hWx\&c=chr1\&o=109399041\& $\mathrm{t}=109401069 \& \mathrm{~g}=\mathrm{mrna} \& \mathrm{i}=\mathrm{AK} 124307)$

5,BC020384(http://www.genome.ucsc.edu/cgi-bin/hgc?hgsid=476093793_1SpaEOeLgD2BNxvEvsDDCbEI8hWx\&c=chr20\&o=63400307\& $\mathrm{t}=63402017 \& \mathrm{~g}=\mathrm{mrna} \& \mathrm{i}=\mathrm{BC} 020384)$

6, AK022024 (http://www.genome.ucsc.edu/cgi-bin/hgc?hgsid=476093793_1SpaEOeLgD2BNxvEvsDDCbEI8hWx\&c=chr4\&o=182531023\& $\mathrm{t}=182533538 \& \mathrm{~g}=\mathrm{mrna} \& \mathrm{i}=\mathrm{AK} 022024)$

7, CR615992 (http://www.ncbi.nlm.nih.gov/nuccore/CR615992.1?report=genbank)

8, AF085995 (http://www.genome.ucsc.edu/cgi-bin/hgc?hgsid=476093793_1SpaEOeLgD2BNxvEvsDDCbEI8hWx\&c=chr4\&o=119512372\& $\mathrm{t}=119552026 \& \mathrm{~g}=\mathrm{mrna} \& \mathrm{i}=\mathrm{AF} 085995)$.

Table II. Univariable and multivariable Cox regression analyses in the training and testing set.

\begin{tabular}{|c|c|c|c|c|c|c|}
\hline \multirow[b]{2}{*}{ Variables } & \multicolumn{3}{|c|}{ Univariable model } & \multicolumn{3}{|c|}{ Multivariable model } \\
\hline & HR & $95 \% \mathrm{CI}$ of $\mathrm{HR}$ & P-value & HR & $95 \% \mathrm{CI}$ of $\mathrm{HR}$ & P-value \\
\hline \multicolumn{7}{|c|}{ Training set (GSE30219) } \\
\hline Risk score & 2.718 & $2.171-3.404$ & $<0.0001$ & 2.666 & $2.108-3.371$ & $<0.0001$ \\
\hline Age (years) & 1.038 & $1.024-1.052$ & $<0.0001$ & 1.028 & $1.013-1.043$ & $<0.0001$ \\
\hline Gender & 0.589 & $0.375-0.926$ & 0.022 & 0.786 & $0.499-1.238$ & 0.299 \\
\hline \multicolumn{7}{|c|}{ Testing set (GSE31210) } \\
\hline Risk score & 2.637 & $1.306-5.326$ & 0.007 & 2.569 & $1.223-5.399$ & 0.013 \\
\hline Age (years) & 1.025 & $0.977-1.075$ & 0.306 & 1.031 & $0.983-1.082$ & 0.211 \\
\hline Gender & 0.658 & $0.338-1.281$ & 0.219 & 0.774 & $0.386-1.553$ & 0.471 \\
\hline \multicolumn{7}{|c|}{ Testing set (GSE37745) } \\
\hline Risk score & 1.667 & $1.138-2.443$ & 0.009 & 1.571 & $1.056-2.338$ & 0.025 \\
\hline Age (years) & 1.011 & $0.994-1.03$ & 0.210 & 1.007 & $0.988-1.025$ & 0.485 \\
\hline Gender & 0.807 & $0.576-1.132$ & 0.214 & 0.888 & $0.628-1.257$ & 0.504 \\
\hline \multicolumn{7}{|c|}{ Testing set (GSE19188) } \\
\hline Risk score & 1.665 & $0.94-2.948$ & 0.081 & 1.42 & $0.782-2.579$ & 0.250 \\
\hline Gender & 0.471 & $0.235-0.943$ & 0.034 & 0.53 & $0.258-1.09$ & 0.084 \\
\hline
\end{tabular}

FDR, false discovery rate; HR, hazards ratio; $\mathrm{CI}$, confidence interval.

patients in the low-risk group (Fig. 3A). The association of the eight-lncRNA risk score and survival was also significant when it was evaluated as a continuous variable in the univariable Cox regression model (Table II). 
A

Enrichment plot: KEGG_CELL_CYCLE

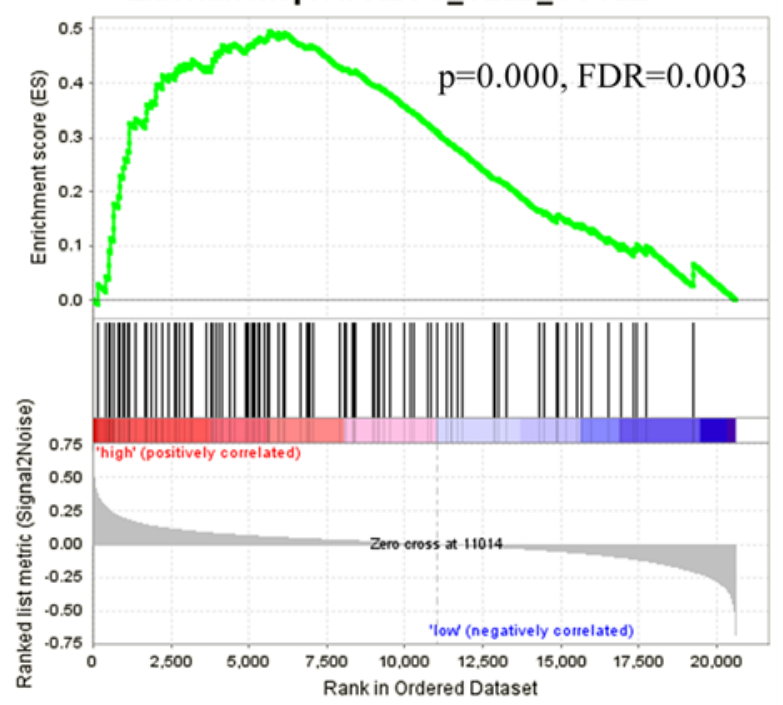

-Enrichment profile - Hits - - Ranking metric scores

C

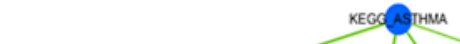

B
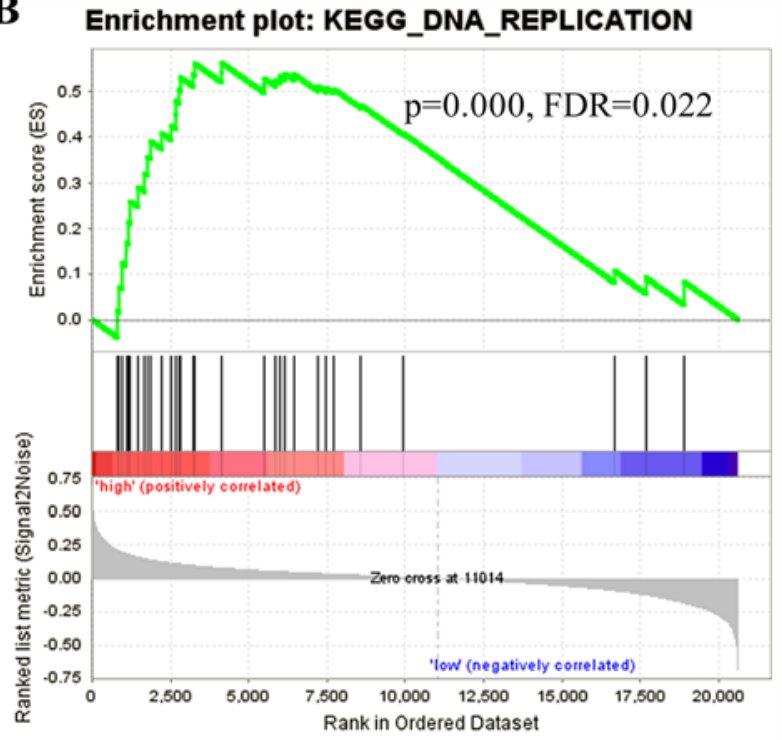

-Enrichment profile — Hits - - Ranking metric scores
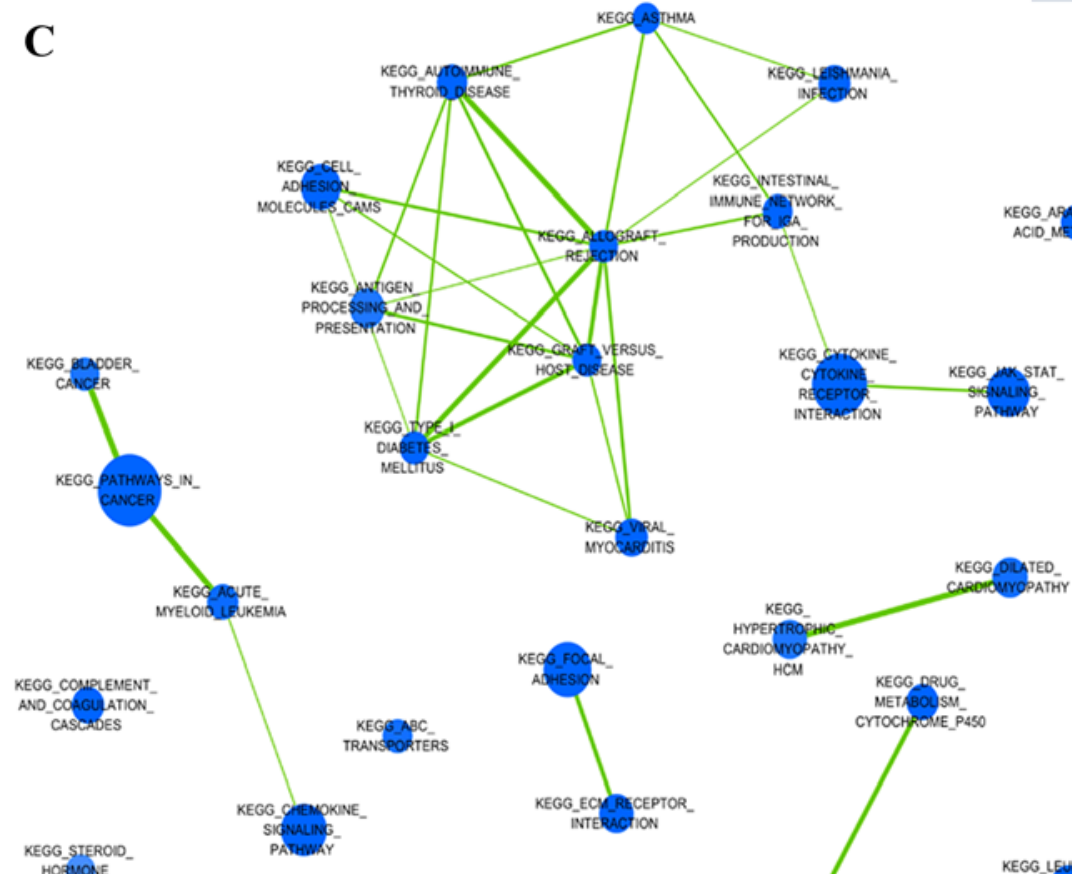

HOF̈NOW.
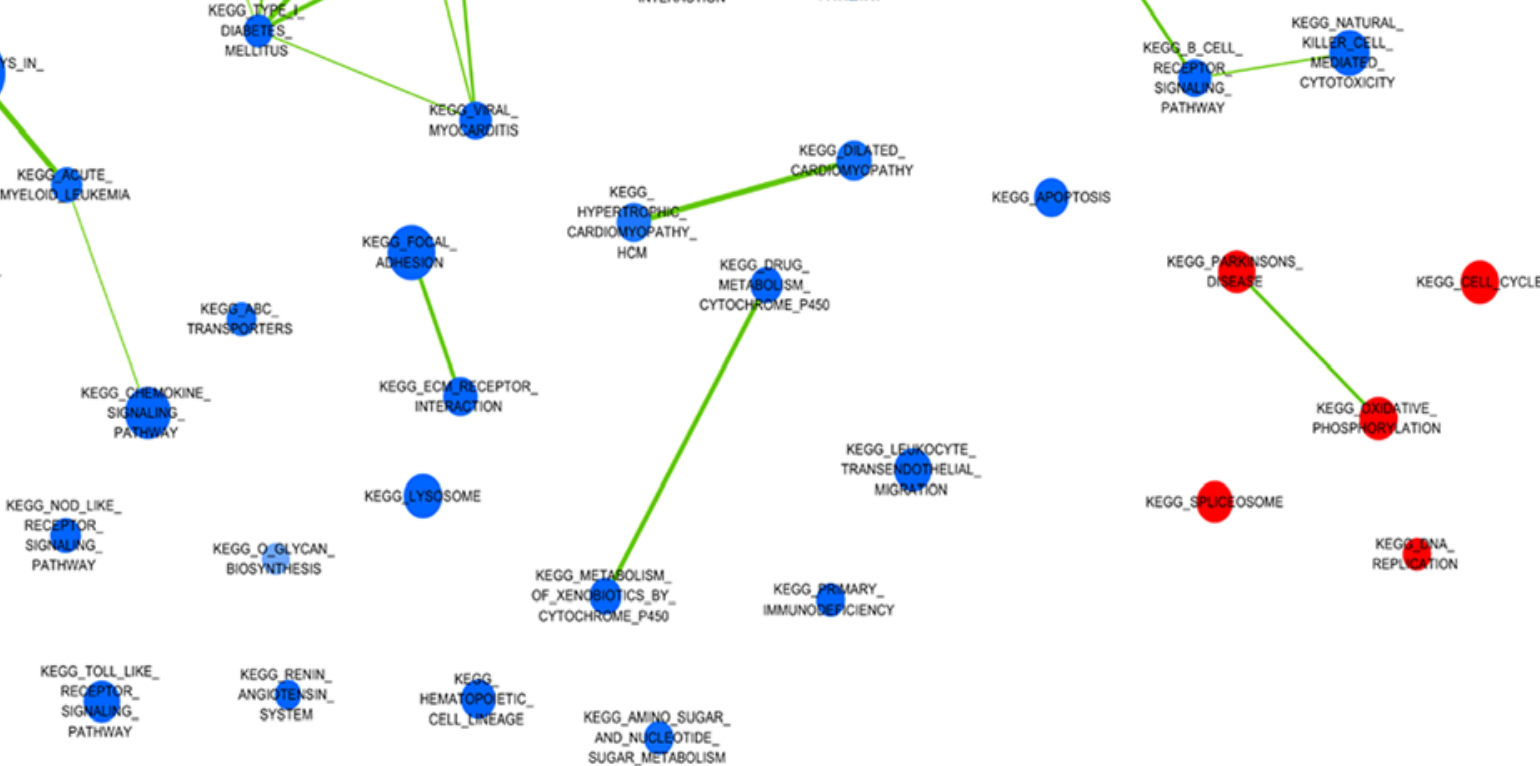

Figure 5. Gene set enrichment analysis delineates biological pathways and processes associated with risk score in the training set (GSE30219). GSEA validated enhanced activity of (A) cell cycle signaling pathway and (B) DNA replication signaling pathway. (C) The functional enrichment map of KEGG terms. Each node represents a KEGG term, which are grouped and annotated by KEGG similarity. A link represents the overlap of shared genes between connecting KEGG terms. Node size represents the number of gene in the KEGG terms.

Validation of the eight-IncRNA signature for survival prediction in the testing sets. To confirm our findings, we calculated the risk score in the testing sets including GSE31210 $(n=226)$, GSE37745 $(n=168)$ and GSE19188 $(n=52)$. Similar to the training set findings, patients in the high-risk group had a shorter survival time than patients in the low-risk group (Fig. 3B-D). Meanwhile, patient survival throughout the follow-up in the low-risk group was better when compared to survival in the high-risk group. In the univariable Cox regression model, the risk score was similar with the high-risk group which had a 
shorter overall survival. The patient survival status (Fig. 4A) and lncRNA values (Fig. 4B) were analyzed independently in the training set. Some of the clinical information (stage and subtype) was not available for a substantial proportion of cases, thus we performed multivariate Cox regression analysis concerning age and gender. The result showed that the eight-lncRNA expression signature was independent of age and gender. Eight-lncRNA risk score, age (available in GSE31210 and GSE37745) and gender (available in GSE31210, GSE37745 and GSE19188) were defined as covariates. These results showed that risk score was an independent predictor of lung cancer patient survival (Table II).

Identification of eight-lncRNA signature-associated biological pathways and processes. GSEA was carried out to identify the associated biological processes and signaling pathways (30). We compared the gene expression profile of lung cancer patients in the low-risk and high-risk groups classified by the eight-lncRNA gene signature in the training set (GSE 30219). The gene sets with significantly different expression (FDR $<0.01 ; \mathrm{p}<0.005)$ were picked up, which implied that the signature may be involved in the cell cycle and DNA replication-related pathways (Fig. 5A and B), and it was visualized as an interaction network with Cytoscape (Fig. 5C). These related pathways were reported to affect cancer cell proliferation (35-37).

\section{Discussion}

As a new class of ncRNAs, lncRNAs were demonstrated to be dysregulated in a variety of diseases, particularly in cancers (38). Numerous studies of abnormal lncRNA expression in various types of cancer suggest that they play an important role in tumorigenesis, and lncRNAs may serve as independent biomarkers for diagnosis and prognosis $(39,40)$. In lung cancer, numerous studies have investigated lncRNAs to predict lung cancer patient survival (41-44). Nevertheless, a single factor to predict the prognosis of tumors is not accurate, since high specificity and sensitivity are lacking for most lncRNAs. Currently, research has found that lncRNA expression profiles can be obtained from publicly available, custom-designed DNA microarrays by re-annotating the array probes $(12,17,34,45,46)$.

In the present study, in order to construct a risk score model, we downloaded four datasets (GSE30219, GSE31210, GSE37745 and GSE19188) from GEO databases, and obtained the IncRNA profiling of lung cancer patients. We identified a prognostic, eight-lncRNA signature from the training set. Furthermore, examination of associated molecular pathways revealed that the eight-lncRNA signature was more likely to involve the cell cycle and DNA replication signaling pathways. Cell cycle disorder and DNA replication induce cell proliferation and affect genome instability, further increasing the possibility of canceration of unstable cells, which participates in tumor occurrence and development $(35,47-49)$. Thus, our findings suggest that lncRNA signatures may provide an efficient classification tool for the clinical prognosis of lung cancer.

Zhou et al (46) also identified an eight-lncRNA signature which may be an effective independent prognostic molecular biomarker in the prediction of non-small lung cancer patient survival, and our findings support the characteristics of the eight-lncRNA. The overexpression of lncRNAs (AK021595, BC030759, AK000053, BC020384 and AK022024) were found to be correlated with shorter survival while other lncRNAs (AK124307, CR615992 and AF085995) were downregulated in the high-risk group compared to the low-risk group. Most importantly, the functional study in cancer of these eight IncRNAs has not been reported to date.

The limitations should be acknowledged for the present study. First, in the present study, only 5,635 (out of 15,000+) human IncRNAs were included. The prognostic lncRNAs identified here may not represent all the lncRNA candidates that were potentially correlated with lung cancer overall survival. Secondly, the longest survival time in the model was 250 months, Thus, the patients in GSE37745 whose survival time was $>300$ months were removed. Thirdly, stage was not included in the present study, since this information was not available for a substantial proportion of cases. Meanwhile, the functions of these eight lncRNAs were inferred by bioinformatics analysis, and these biological roles in tumorigenesis were not clear and should be investigated in experimental studies.

In summary, we identified a signature of a set of eight IncRNAs, which predicted the overall survival in three independent testing sets. Further bioinformatic analysis revealed that the prognostic value was independent of age and gender. Moreover, these lncRNAs are involved in cell cycle and DNA replication signaling pathways. These lncRNAs may have clinical implications as diagnostic markers. However, the biological roles of these eight lncRNAs in tumorigenesis require further study.

\section{Acknowledgements}

The present study was supported by the National Natural Science Foundation of China (nos. 81400121, 81270607, 81541027 and 81501352).

\section{References}

1. Jemal A, Siegel R, Xu J and Ward E: Cancer statistics, 2010. CA Cancer J Clin 60: 277-300, 2010.

2. Torre LA, Bray F, Siegel RL, Ferlay J, Lortet-Tieulent J and Jemal A: Global cancer statistics, 2012. CA Cancer J Clin 65: 87-108, 2015

3. DeSantis CE, Lin CC, Mariotto AB, Siegel RL, Stein KD, Kramer JL, Alteri R, Robbins AS and Jemal A: Cancer treatment and survivorship statistics, 2014. CA Cancer J Clin 64: 252-271, 2014.

4. Ling H, Vincent K, Pichler M, Fodde R, Berindan-Neagoe I, Slack FJ and Calin GA: Junk DNA and the long non-coding RNA twist in cancer genetics. Oncogene 34: 5003-5011, 2015.

5. Lipovich L, Johnson R and Lin CY: MacroRNA underdogs in a microRNA world: Evolutionary, regulatory, and biomedical significance of mammalian long non-protein-coding RNA. Biochim Biophys Acta 1799: 597-615, 2010.

6. Fatica A and Bozzoni I: Long non-coding RNAs: New players in cell differentiation and development. Nat Rev Genet 15: 7-21, 2014.

7. Qi P and Du X: The long non-coding RNAs, a new cancer diagnostic and therapeutic gold mine. Mod Pathol 26: 155-165, 2013.

8. Yarmishyn AA, Batagov AO, Tan JZ, Sundaram GM, Sampath P Kuznetsov VA and Kurochkin IV: HOXD-AS1 is a novel lncRNA encoded in HOXD cluster and a marker of neuroblastoma progression revealed via integrative analysis of noncoding transcriptome. BMC Genomics 15 (Suppl 9): S7, 2014. 
9. Crea F, Watahiki A, Quagliata L, Xue H, Pikor L, Parolia A, Wang Y, Lin D, Lam WL, Farrar WL, et al: Identification of a long non-coding RNA as a novel biomarker and potential therapeutic target for metastatic prostate cancer. Oncotarget 5: 764-774, 2014.

10. Shen X, Xie B, Ma Z, Yu W, Wang W, Xu D, Yan X, Chen B, Yu L, Li J, et al: Identification of novel long non-coding RNAs in triple-negative breast cancer. Oncotarget 6: 21730-21739, 2015.

11. Sun J, Chen X, Wang Z, Guo M, Shi H, Wang X, Cheng L and Zhou M: A potential prognostic long non-coding RNA signature to predict metastasis-free survival of breast cancer patients. Sci Rep 5: 16553, 2015.

12. Meng J, Li P, Zhang Q, Yang Z and Fu S: A four-long non-coding RNA signature in predicting breast cancer survival. J Exp Clin Cancer Res 33: 84, 2014.

13. Zhou M, Wang X, Shi H, Cheng L, Wang Z, Zhao H, Yang L and Sun J: Characterization of long non-coding RNA-associated ceRNA network to reveal potential prognostic lncRNA biomarkers in human ovarian cancer. Oncotarget: Feb 3, 2016 (Epub ahead of print). doi: 10.18632/oncotarget.7181.

14. Malek E, Jagannathan S and Driscoll JJ: Correlation of long non-coding RNA expression with metastasis, drug resistance and clinical outcome in cancer. Oncotarget 5: 8027-8038, 2014.

15. Chen F, Tian Y, Pang EJ, Wang Y and Li L: MALAT2-activated long noncoding RNA indicates a biomarker of poor prognosis in gastric cancer. Cancer Gene Ther: Feb 27, 2015 (Epub ahead of print). doi: 10.1038/cgt.2015.6.

16. Xue Y, Ma G, Gu D, Zhu L, Hua Q, Du M, Chu H, Tong N, Chen J, Zhang Z, et al: Genome-wide analysis of long noncoding RNA signature in human colorectal cancer. Gene 556: 227-234, 2015.

17. Hu Y, Chen HY, Yu CY, Xu J, Wang JL, Qian J, Zhang X and Fang JY: A long non-coding RNA signature to improve prognosis prediction of colorectal cancer. Oncotarget 5: 2230-2242, 2014.

18. Zhou M, Zhao H, Wang Z, et al: Identification and validation of potential prognostic lncRNA biomarkers for predicting survival in patients with multiple myeloma. J Exp Clin Cancer Res 34: $102,2015$.

19. Rousseaux S, Debernardi A, Jacquiau B, Vitte AL, Vesin A, Nagy-Mignotte H, Moro-Sibilot D, Brichon PY, Lantuejoul S, Hainaut $\mathrm{P}$, et al: Ectopic activation of germline and placental genes identifies aggressive metastasis-prone lung cancers. Sci Transl Med 5: 186ra66, 2013.

20. Yamauchi M, Yamaguchi R, Nakata A, Kohno T, Nagasaki M, Shimamura T, Imoto S, Saito A, Ueno K, Hatanaka Y, et al: Epidermal growth factor receptor tyrosine kinase defines critical prognostic genes of stage I lung adenocarcinoma. PLoS One 7: e43923, 2012.

21. Botling J, Edlund K, Lohr M, Hellwig B, Holmberg L, Lambe M, Berglund A, Ekman S, Bergqvist M, Pontén F, et al: Biomarker discovery in non-small cell lung cancer: Integrating gene expression profiling, meta-analysis, and tissue microarray validation. Clin Cancer Res 19: 194-204, 2013.

22. Hou J, Aerts J, den Hamer B, van Ijcken W, den Bakker M, Riegman P, van der Leest C, van der Spek P, Foekens JA, Hoogsteden HC, et al: Gene expression-based classification of non-small cell lung carcinomas and survival prediction. PLoS One 5: e10312, 2010.

23. Irizarry RA, Bolstad BM, Collin F, Cope LM, Hobbs B and Speed TP: Summaries of Affymetrix GeneChip probe level data Nucleic Acids Res 31: e15, 2003.

24. Risueño A, Fontanillo C, Dinger ME and De Las Rivas J: GATExplorer: Genomic and transcriptomic explorer; mapping expression probes to gene loci, transcripts, exons and ncRNAs. BMC Bioinformatics 11: 221, 2010.

25. Pang KC, Stephen S, Engström PG, Tajul-Arifin K, Chen W, Wahlestedt C, Lenhard B, Hayashizaki Y and Mattick JS: RNAdb - a comprehensive mammalian noncoding RNA database. Nucleic Acids Res 33: D125-D130, 2005.

26. Sun L, Luo H, Bu D, Zhao G, Yu K, Zhang C, Liu Y, Chen R and Zhao Y: Utilizing sequence intrinsic composition to classify protein-coding and long non-coding transcripts. Nucleic Acids Res 41: e166, 2013.

27. Simon R, Lam A, Li MC, Ngan M, Menenzes S and Zhao Y: Analysis of gene expression data using BRB-ArrayTools. Cancer Inform 3: 11-17, 2007.
28. Ishwaran $\mathrm{H}$ and Kogalur UB: Consistency of random survival forests. Stat Probab Lett 80: 1056-1064, 2010.

29. Bralten LB and French PJ: Genetic alterations in glioma. Cancers 3: 1129-1140, 2011

30. Subramanian A, Tamayo P, Mootha VK, Mukherjee S, Ebert BL, Gillette MA, Paulovich A, Pomeroy SL, Golub TR, Lander ES, et al: Gene set enrichment analysis: A knowledge-based approach for interpreting genome-wide expression profiles. Proc Natl Acad Sci USA 102: 15545-15550, 2005.

31. Merico D, Isserlin R, Stueker O, Emili A and Bader GD: Enrichment map: A network-based method for gene-set enrichment visualization and interpretation. PLoS One 5: e13984, 2010.

32. Huang W, Sherman BT and Lempicki RA: Bioinformatics enrichment tools: Paths toward the comprehensive functional analysis of large gene lists. Nucleic Acids Res 37: 1-13, 2009.

33. Dinger ME, Pang KC, Mercer TR, Crowe ML, Grimmond SM and Mattick JS: NRED: A database of long noncoding RNA expression. Nucleic Acids Res 37: D122-D126, 2009.

34. Du Z, Fei T, Verhaak RG, Su Z, Zhang Y, Brown M, Chen Y and Liu XS: Integrative genomic analyses reveal clinically relevant long noncoding RNAs in human cancer. Nat Struct Mol Biol 20: 908-913, 2013

35. Williams GH and Stoeber K: The cell cycle and cancer. J Pathol 226: 352-364, 2012.

36. Kim T, Jeon YJ, Cui R, Lee JH, Peng Y, Kim SH, Tili E, Alder H and Croce CM: Role of MYC-regulated long noncoding RNAs in cell cycle regulation and tumorigenesis. J Natl Cancer Inst 107: pii: dju505, 2015.

37. Macheret $M$ and Halazonetis TD: DNA replication stress as a hallmark of cancer. Annu Rev Pathol 10: 425-448, 2015.

38. Yang G, Lu X and Yuan L: LncRNA: A link between RNA and cancer. Biochim Biophys Acta 1839: 1097-1109, 2014.

39. Li J, Chen Z, Tian L, Zhou C, He MY, Gao Y, Wang S, Zhou F, Shi S, Feng X, et al: LncRNA profile study reveals a threelncRNA signature associated with the survival of patients with oesophageal squamous cell carcinoma. Gut 63: 1700-1710, 2014.

40. Fernando TR, Rodriguez-Malave NI, Waters EV, Yan W, Casero D, Basso G, Pigazzi M and Rao DS: LncRNA expression discriminates karyotype and predicts survival in B-lymphoblastic leukemia. Mol Cancer Res 13: 839-851, 2015.

41. Yang YR, Zang SZ, Zhong CL, Li YX, Zhao SS and Feng XJ: Increased expression of the IncRNA PVT1 promotes tumorigenesis in non-small cell lung cancer. Int J Clin Exp Pathol 7: 6929-6935, 2014.

42. Xie X, Liu HT, Mei J, Ding FB, Xiao HB, Hu FQ, Hu R and Wang MS: LncRNA HMlincRNA717 is down-regulated in non-small cell lung cancer and associated with poor prognosis. Int J Clin Exp Pathol 7: 8881-8886, 2014.

43. Han L, Zhang EB, Yin DD, Kong R, Xu TP, Chen WM, Xia R, Shu YQ and De W: Low expression of long noncoding RNA PANDAR predicts a poor prognosis of non-small cell lung cancer and affects cell apoptosis by regulating Bcl-2. Cell Death Dis 6: e1665, 2015.

44. Lin L, Gu ZT, Chen WH and Cao KJ: Increased expression of the long non-coding RNA ANRIL promotes lung cancer cell metastasis and correlates with poor prognosis. Diagn Pathol 10: 14, 2015.

45. Zhang X, Sun S, Pu JK, Tsang AC, Lee D, Man VO, Lui WM, Wong ST and Leung GK: Long non-coding RNA expression profiles predict clinical phenotypes in glioma. Neurobiol Dis 48: $1-8,2012$.

46. Zhou M, Guo M, He D, Wang X, Cui Y, Yang H, Hao D and Sun J: A potential signature of eight long non-coding RNAs predicts survival in patients with non-small cell lung cancer. J Transl Med 13: 231, 2015.

47. Desjobert C,El Maï M, Gérard-Hirne T, Guianvarc'h D, Carrier A, Pottier C, Arimondo PB and Riond J: Combined analysis of DNA methylation and cell cycle in cancer cells. Epigenetics 10: 82-91, 2015.

48. Tachibana KE, Gonzalez MA and Coleman N: Cell-cycledependent regulation of DNA replication and its relevance to cancer pathology. J Pathol 205: 123-129, 2005.

49. Falaschi A, Abdurashidova $G$ and Biamonti G: DNA replication, development and cancer: A homeotic connection? Crit Rev Biochem Mol Biol 45: 14-22, 2010. 